\title{
Pion observables within the covariant formulation of Light-front dynamics
}

\author{
Natalia Tsirova* \\ Clermont Université, Laboratoire de Physique Corpusculaire, BP10448, F-63000 \\ Clermont-Ferrand, France \\ E-mail: natalia.tsirova@clermont.in2p3.fr

\section{Olivier Leitner} \\ Université Pierre et Marie Curie, LPNHE, F-75252, Paris, France \\ E-mail: leitneralpnhe.in2p3.fr

\section{Jean-François Mathiot} \\ Clermont Université, Laboratoire de Physique Corpusculaire, BP10448, F-63000 \\ Clermont-Ferrand, France \\ E-mail: mathioteclermont.in2p3.fr
}

\begin{abstract}
Within the covariant formulation of light-front dynamics we determine the two spin components of the pion wave function in a phenomenological constituent quark model. These spin components are further interpreted as a non-relativistic wave function and a purely relativistic component. The latter is determined in the one-gluon exchange approximation. We calculate in this model several observables: the pion decay constant, the electromagnetic form factor and the transition form factor.
\end{abstract}

Light Cone 2010 - LC2010

June 14-18, 2010

Valencia, Spain

*Speaker. 


\section{Covariant formulation of Light-front dynamics}

For many years the knowledge and description of the structure of elementary particles have been of great interest in particle physics. Many theoretical frameworks already exist (QCD sum rules, lattice QCD, etc.), all of them have their intrinsic theoretical limitations. In order to have more physical insights into the internal structure of hadrons we have thus still to rely on constituent quark models. For the pion description these models should be relativistic.

The first requirement to build a relativistic dynamical theory is that it should be invariant under the ten generators of the Poincaré group. Following this requirement three forms of dynamics have been derived by Dirac: the instant form, the point form and the front form. We will concentrate in this study on the front form, namely on its explicitly covariant form (CLFD) [1]. The orientation of the light front plane is here characterized by an arbitrary light like four vector $\omega$ with $\omega \cdot x=c t e$ and $\omega^{2}=0$. This approach is a generalization of the standard light-front dynamics (LFD) [2]. The latter can easily be recovered with a special choice of the light-front orientation, $\omega=(1,0,0,-1)$.

The two-body wave function $\Phi_{\sigma_{1} \sigma_{2}}^{\lambda}$ can be parametrized in terms of various sets of kinematical variables. Here $\lambda$ is the projection of the total angular momentum of the system on the $z$ axis in the rest frame and $\sigma_{i}$ is the spin projections of the particle $i$ in the corresponding rest system. In order to make a close connection to the non-relativistic case, it is convenient to introduce the following variables [3] defined by

$$
\begin{gathered}
\mathbf{k}=L^{-1}(\mathscr{P}) \mathbf{k}_{1}=\mathbf{k}_{1}-\frac{\mathscr{P}}{\sqrt{\mathscr{P}^{2}}}\left[k_{10}-\frac{\mathbf{k}_{1} \cdot \mathscr{P}}{\sqrt{\mathscr{P}^{2}}+\mathscr{P}_{0}}\right], \\
\mathbf{n}=\frac{L^{-1}(\mathscr{P}) \omega}{\left|L^{-1}(\mathscr{P}) \omega\right|}=\sqrt{\mathscr{P}^{2}} \frac{L^{-1}(\mathscr{P}) \omega}{\omega \cdot p},
\end{gathered}
$$

where $L^{-1}(\mathscr{P})$ is the (inverse) Lorentz boost of momentum $\mathscr{P}$. We denote by $k_{1}\left(k_{2}\right)$ the momentum of the constituent antiquark (quark). The momentum $\mathbf{k}$ corresponds, in the frame where $\mathbf{k}_{1}+\mathbf{k}_{2}=\mathbf{0}$, to the usual relative momentum between the two particles. The unit vector $\mathbf{n}$ corresponds, in this frame, to the spatial direction of $\omega$. The second set of variables we shall also use in the following is the usual light-front set of coordinates $\left(x, \mathbf{R}_{\perp}\right)$ which is defined by

$$
x=\frac{\omega \cdot k_{1}}{\omega \cdot p}, \quad R_{1}=k_{1}-x p
$$

where $R_{1}$ is decomposed into its spatial components parallel and perpendicular to the direction of the light-front, $R_{1}=\left(R_{0}, \mathbf{R}_{\perp}, \mathbf{R}_{\|}\right)$. We have by definition $R_{1} \cdot \omega=0$, and thus $R_{1}^{2}=-\mathbf{R}_{\perp}^{2}$. In the reference frame where $\mathbf{p}_{\perp}=0, \mathbf{R}_{\perp}$ is identical to the usual transverse momentum $\mathbf{k}_{\perp}$.

\section{The pion wave function}

The covariance of our approach allows to write down explicitly the general spin structure of the two-body bound state. For a pseudoscalar particle of momentum $p$, composed of an antiquark and a quark of equal mass $m$ it takes the form

$$
\Phi_{\sigma_{1} \sigma_{2}}^{\lambda=0}=\frac{1}{\sqrt{2}} \bar{u}_{\sigma_{2}}\left(k_{2}\right)\left(A_{1} \frac{1}{m}+A_{2} \frac{\phi}{\omega \cdot p}\right) \gamma_{5} v_{\sigma_{1}}\left(k_{1}\right)
$$


where $v\left(k_{1}\right)$ and $\bar{u}\left(k_{2}\right)$ are the usual Dirac spinors, and $A_{1}$ and $A_{2}$ are the two scalar components of the pion wave function. For simplicity, we shall also call wave functions these two spin components. They depend on two scalar variables, which we shall choose as $\left(x, \mathbf{R}_{\perp}^{2}\right)$. The representation of this wave function in terms of the variables $\mathbf{k}$ and $\mathbf{n}$ is given by

$$
\Phi_{\sigma_{1} \sigma_{2}}^{0}=\frac{1}{\sqrt{2}} w_{\sigma_{2}}^{t}\left(g_{1}+\frac{i \sigma \cdot[\mathbf{n} \times \mathbf{k}]}{k} g_{2}\right) w_{\sigma_{1}}
$$

where $w_{i}$ are Pauli spinors and $g_{1,2}$ are the two scalar components of the pion wave function in this representation. One can easily express $A_{1,2}$ in terms of $g_{1,2}$. We get

$$
g_{1}=\frac{2 \varepsilon_{k}}{m} A_{1}+\frac{m}{\varepsilon_{k}} A_{2}, g_{2}=-\frac{k}{\varepsilon_{k}} A_{2} .
$$

We would like to stress that the decomposition (2.1) is a very general one for a spin zero particle composed of two spin $1 / 2$ constituents. In the non-relativistic limit, $\mathbf{n} \equiv \frac{\mathbf{n}}{c} \rightarrow 0$ and the component $g_{1}\left(\mathbf{k}^{2}, \mathbf{k} . \mathbf{n}\right)$ only survives and depends on a single scalar variable $\mathbf{k}^{2}$. In our phenomenological analysis, we shall therefore start from a non-relativistic component, $g_{1}^{0}$, given by a simple parametrization. We shall use in the following either a gaussian wave function given by

$$
g_{1}^{0}\left(\mathbf{k}^{2}\right)=\alpha \exp \left(-\beta \mathbf{k}^{2}\right)
$$

or a power-law wave function written as

$$
g_{1}^{0}\left(\mathbf{k}^{2}\right)=\frac{\alpha}{\left(1+\beta \mathbf{k}^{2}\right)^{2}},
$$

where $\alpha$ and $\beta$ are two parameters to be determined from experimental data and the condition of normalization. The latter writes [3]

$$
1=\sum_{\sigma_{1} \sigma_{2}} \int d D \Phi_{\sigma_{1} \sigma_{2}}^{\lambda} \Phi_{\sigma_{1} \sigma_{2}}^{\lambda \star}
$$

where $d D$ is an invariant phase space element which can take the following forms, depending on the kinematical variables which are used

$$
d D=\frac{1}{(2 \pi)^{3}} \frac{\mathrm{d}^{3} \mathbf{k}_{1}}{(1-x) 2 \varepsilon_{k_{1}}}=\frac{1}{(2 \pi)^{3}} \frac{\mathrm{d}^{3} \mathbf{k}}{2 \varepsilon_{k}}=\frac{1}{(2 \pi)^{3}} \frac{\mathrm{d}^{2} \mathbf{R}_{\perp} \mathrm{d} x}{2 x(1-x)} .
$$

It is necessary to correct the nonrelativistic component in some way in order to incorporate in a full relativistic framework the high momentum tail given by the one-gluon exchange mechanism. We shall achieve this using perturbation theory, starting from the zeroth order wave function $g_{1}^{0}$.

The (eigenvalue) equation we start from to calculate the bound state wave function is represented schematically in Fig. (1). This equation writes, in the case of spin 1/2 particles [3]

$$
\begin{aligned}
& \bar{u}\left(k_{2}\right) \Gamma_{2} v\left(k_{1}\right)=\int \frac{\mathrm{d}^{3} \mathbf{k}_{1}}{2 \varepsilon_{k_{1}}(2 \pi)^{3}} \frac{\mathrm{d} \tau^{\prime}}{\tau^{\prime}-i \varepsilon} \delta\left(k_{2}^{\prime 2}-m^{2}\right) \Theta\left(\omega \cdot k_{2}^{\prime}\right) \\
& \times \bar{u}\left(k_{2}\right)\left[\gamma_{\mu}\left(\not k_{2}^{\prime}+m\right) \Gamma_{2}^{\prime}\left(m-\not k_{1}^{\prime}\right) \gamma_{v}\right] K^{\mu v} v\left(k_{1}\right) .
\end{aligned}
$$

It is written in terms of the two-body vertex function $\Gamma_{2}$ defined by [4]

$$
\bar{u}_{\sigma_{2}}\left(k_{2}\right) \Gamma_{2} v_{\sigma_{1}}\left(k_{1}\right) \equiv\left(s-M_{\pi}^{2}\right) \Phi_{\sigma_{1} \sigma_{2}}^{0},
$$




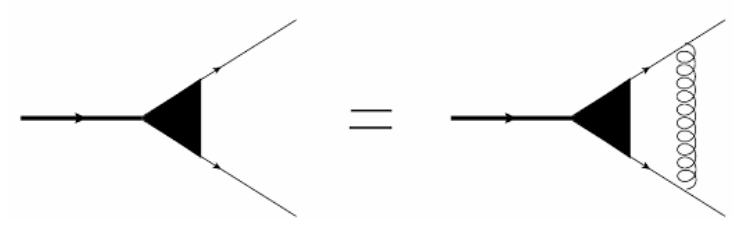

Figure 1: Calculation of radiative corrections to the two-body wave function.

with $s=\frac{\mathbf{R}_{\perp}^{2}+m^{2}}{x}+\frac{\mathbf{R}_{\perp}^{2}+m^{2}}{(1-x)}$. The mass of the pion is denoted by $M_{\pi}$.

The kernel, $K^{\mu v}$, including the appropriate color factor, can be written as $K^{\mu v}=-g^{\mu v} \frac{4}{3} g^{2} \mathscr{K}$ in the Feynman gauge, where $\mathscr{K}$ is the usual one-gluon exchange kernel. The quark gluon coupling constant is denoted by $g$, with $g^{2}=4 \pi \alpha_{s}$. In order to incorporate the correct short range properties of the quark-antiquark interaction from asymptotic freedom, we shall consider in the following a running coupling constant $\alpha_{s}\left(K^{2}\right)$, where $K^{2}$ is the off-shell momentum squared of the gluon. It is given by $K^{2}=1 / \mathscr{K}$. We choose a simple parametrization which gives, in the large $K^{2}$ limit, the known behavior given by perturbative QCD. We take

$$
\alpha_{s}\left(K^{2}\right)=\frac{\alpha_{s}^{0}}{1+\frac{11-\frac{2}{3} n_{f}}{4 \pi} \alpha_{s}^{0} \log \left[\frac{\left|K^{2}\right|+\Lambda_{Q C D}^{2}}{\Lambda_{Q C D}^{2}}\right]} .
$$

At small $K^{2}$, it is given by the parameter $\alpha_{s}^{0}$ which should be of the order of 1 . We choose $n_{f}=2$ and $\Lambda_{Q C D}=220 \mathrm{MeV}$.

In order to calculate the relativistic corrections we proceed as follows. We substitute the nonrelativistic components to the r.h.s. of (2.8) They are calculated from (2.4) or (2.5) by means of (2.3) with $g_{1}=g_{1}^{0}$ and $g_{2}=g_{2}^{0}=0$. Then we solve (2.8) and obtain the relativistic components $\delta A_{1,2}$ substituted to the 1.h.s. The total components are then given by

$$
A_{1,2}=A_{1,2}^{0}+\delta A_{1,2}
$$

\section{Physical observables}

The aim of our work is to get an overall good description of the following pion observables: pion decay constant, electromagnetic and transition form factors and charge radius.

\subsection{Decay constant}

The pseudoscalar decay amplitude is given by the diagram in Fig. (2). Using the diagrammatic

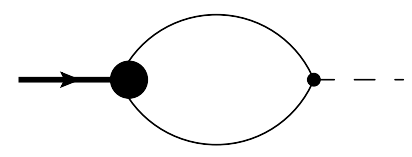

Figure 2: Decay amplitude of the pion. 
rules of CLFD [3], we can calculate the decay constant $f_{\pi}$ from the graph indicated in Fig. (2). One gets in terms of the functions $A$, including color factors,

$$
f_{\pi}=\frac{2 \sqrt{6}}{(2 \pi)^{3}} \int \frac{\mathrm{d}^{2} \mathbf{R}_{\perp} \mathrm{d} x}{2 x(1-x)}\left[A_{1}+2 x(1-x) A_{2}\right] .
$$

The pion decay constant is divergent like $\log \log \mathbf{R}_{\perp}^{2} / \Lambda_{Q C D}^{2}$. This divergence is extremely soft. To get the physical contribution, we just subtract the minimal contribution arising when the integral on $\left|\mathbf{R}_{\perp}\right|$ is cut-off to $\Lambda_{C}$.

\subsection{Electromagnetic form factor}

This physical observable is very powerful in order to constrain the phenomenological structure of the wave function both in the low and high momentum scales. In the impulse approximation, the electromagnetic form factor is shown in Fig. (3).

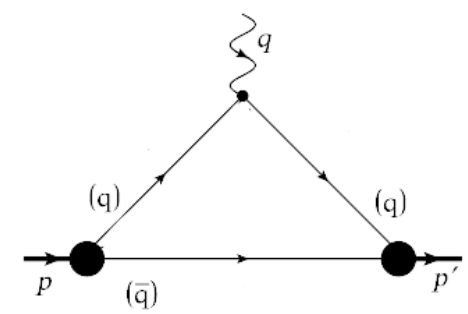

Figure 3: Pion electromagnetic form factor in the impulse approximation. A similar contribution where the photon couples to the antiquark is not shown for simplicity.

By using the diagrammatic rules of CLFD, we can write down the electromagnetic amplitude corresponding to Fig. (3) where the photon interacts with the quark. Assuming it is pointlike, one obtains:

$$
F_{\pi}^{\gamma q}\left(Q^{2}\right)=\frac{e_{q}}{(2 \pi)^{3}} \int \frac{\mathrm{d}^{2} \mathbf{R}_{\perp} \mathrm{d} x}{2 x(1-x)}\left[\frac{m^{2}+\mathbf{R}_{\perp}^{2}-x \mathbf{R}_{\perp} \cdot \Delta}{x(1-x) m^{2}} A_{1} A_{1}^{\prime}+2\left(A_{1} A_{2}^{\prime}+A_{1}^{\prime} A_{2}\right)+4 x(1-x) A_{2} A_{2}^{\prime}\right]
$$

If we define the four momentum transfer $q$ by $q=\left(q_{0}, \Delta, \mathbf{q}_{\|}\right)$, with $\Delta \cdot \omega=0$ and $\mathbf{q}_{\|}$parallel to $\omega$, we have $Q^{2}=-q^{2} \equiv \Delta^{2}$, and thus $\mathbf{R}_{\perp}^{\prime}=\mathbf{R}_{\perp}-x \Delta$. The contribution from the coupling of the photon to the antiquark can be deduced from (3.2) by the interchange $x \Longleftrightarrow(1-x), \mathbf{R}_{\perp} \Longleftrightarrow-\mathbf{R}_{\perp}$ and an overall change of sign. One thus obtains the full contribution to the electromagnetic form factor of the pion

$$
F_{\pi}\left(Q^{2}\right)=F_{\pi}^{\gamma q}\left(Q^{2}\right)+F_{\pi}^{\gamma \bar{q}}\left(Q^{2}\right)
$$

Note that this form factor, in the impulse approximation, is completely finite since it does not correspond to any radiative corrections at the $\gamma q$ vertex. The charge radius of the pion, $\left\langle r_{\pi}^{2}\right\rangle^{1 / 2}$, can be extracted from $F_{\pi}\left(Q^{2}\right)$ according to

$$
\left\langle r_{\pi}^{2}\right\rangle=-\left.6 \frac{\mathrm{d}}{\mathrm{d} Q^{2}} F_{\pi}\left(Q^{2}\right)\right|_{Q^{2}=0} .
$$




\subsection{Transition form factor}

For the transition form factor in the impulse approximation, the first relevant diagram, where the virtual photon couples to the quark, is indicated in Fig. (4). The second contribution involving

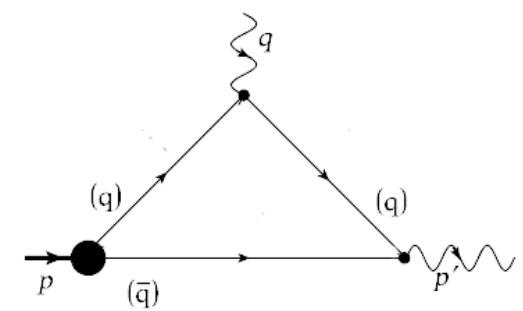

Figure 4: Pion transition form factor in the impulse approximation. A similar contribution where the virtual photon couples to the antiquark is not shown for simplicity.

the coupling of the virtual photon to the antiquark can be calculated similarly. The total amplitude for the transition form factor reads

$$
\begin{aligned}
F_{\pi \gamma}\left(Q^{2}\right) & =\frac{4 \sqrt{3}\left(e_{u}^{2}-e_{d}^{2}\right)}{(2 \pi)^{3}} \int \frac{\mathrm{d}^{2} \mathbf{R}_{\perp} \mathrm{d} x}{2 x(1-x)} \frac{x}{m^{2}+\mathbf{R}_{\perp}^{2}-2 \mathbf{R}_{\perp} \cdot \Delta+x^{2} Q^{2}} \\
& \times\left[A_{1}+2 x(1-x) A_{2}-\frac{\mathbf{R}_{\perp} \cdot \Delta}{Q^{2}}(1-x) A_{2}\right] .
\end{aligned}
$$

The transition form factor of the pion is completely finite thanks to the extra dependence on the transverse momentum as compared to the decay constant (3.1).

\section{Numerical results}

Our phenomenological analysis has three independent parameters. The first one, $\beta$, gives the typical size of the non-relativistic wave function we start from in $(2.4,2.5)$. The second parameter is the quark (or antiquark) constituent mass $m$. The third one is the strong coupling constant in the low momentum region given by $\alpha_{s}^{0}$ in (2.10). The values of these parameters are indicated in Table 1 , for the two types of non-relativistic wave functions used in this study.

\begin{tabular}{c|ccc}
\hline \hline & $\beta$ & $m$ & $\alpha_{s}^{0}$ \\
\hline Gauss w.f. & 3.5 & $250 \mathrm{MeV}$ & 1.3 \\
Power-law w.f. & 3.72 & $250 \mathrm{MeV}$ & 0.35 \\
\hline \hline
\end{tabular}

Table 1: Parameter sets of the calculation.

These three parameters are fixed to get an overall good description of the pion decay constant, charge radius, electromagnetic and transition form factors. Our predictions for the pion decay constant and charge radius are in excellent agreement with the experimental data: $f_{\pi}=131 \mathrm{MeV}$, $\left\langle r_{\pi}^{2}\right\rangle^{1 / 2}=0.67 \mathrm{MeV}$ for both wave functions. 
The pion electromagnetic and transition from factors are shown in Figs. (5-6) for the two types of wave functions used in this study. Given the large experimental errors at large momentum transfer, we do not attempt in this study to get a best fit to all the data, but just to show that an overall agreement of all the available data is possible within our framework.

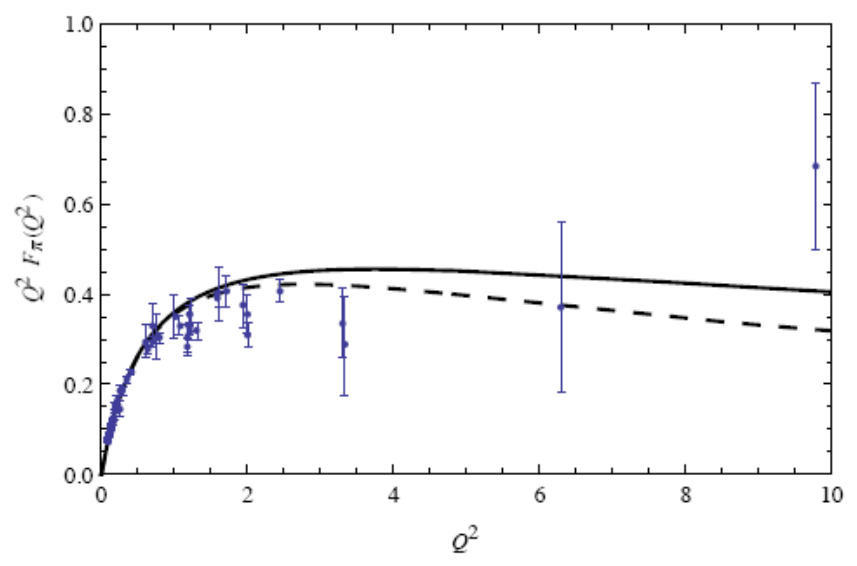

Figure 5: Pion electromagnetic form factor calculated with two types of wave function. The solid (dashed) line corresponds to the power-law (gaussian) wave function in the non-relativistic limit. The experimental data are from $[5,6,7,8,9,10,11,12]$.

The pion electromagnetic form factor is shown in Fig. (5) together with the world wide experimental data. Given the experimental errors which are large above $3 \mathrm{GeV}^{2}$, both parametrization (gaussian or power-law) give a rather good account of the data, in the whole kinematical domain available.

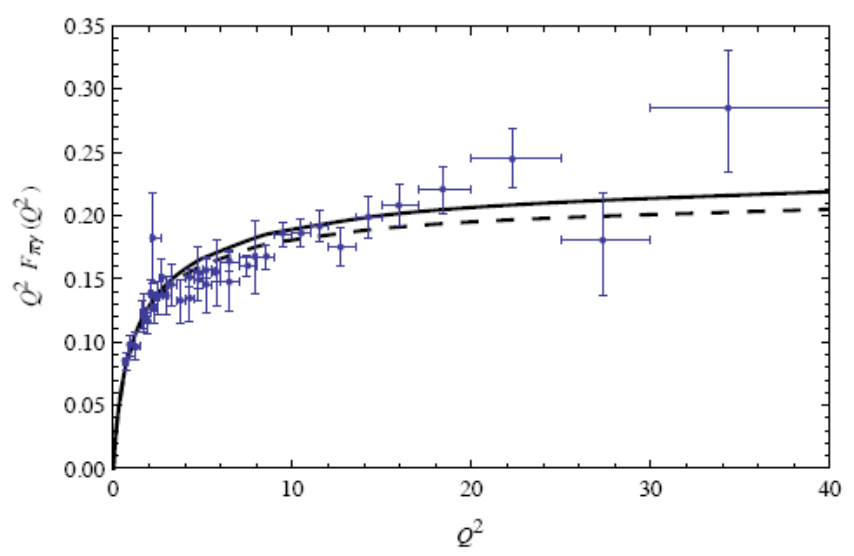

Figure 6: Pion transition form factor calculated with two types of wave function. The solid (dashed) line corresponds to the power-law (gaussian) wave function in the non-relativistic limit. The experimental data are from $[13,14,15]$.

The corresponding results for the pion transition form factor are shown in Fig. (6). Both parametrizations are also in good agreement with the experimental data. At very high momentum transfer however, for $Q^{2}>15 \mathrm{GeV}^{2}$, our results underestimate slightly the experimental data. There 
is no way to adjust our parameters to get a better agreement for the transition form factor without spoiling the good agreement we get for the electromagnteic form factor. We should however wait for more precise experimental data before drawing any definite conclusions.

\section{Summary}

We have investigated in this study the full relativistic structure of the pion in the framework of the constituent quark model. Our wave function is constructed starting from a purely phenomenological wave function in the non relativistic limit. Dynamical relativistic corrections are included by a one gluon exchange process. This latter generates the necessary relativistic high momentum components in the pion wave function.

From this full structure of the pion wave function, we have been able to have an overall very good agreement with all experimental data available, both in the low and high momentum domain. It is necessary to confirm the recent Babar data for the pion transition form factor at very high momentum transfer (till about $Q^{2} \simeq 40 \mathrm{GeV}^{2}$ ), with more precise data.

This analysis shows also the real flexibility of CLFD in describing few body systems in relativistic nuclear and particle physics. Its application to more fundamental calculations starting from first principles is also under way [16].

\section{References}

[1] V.A. Karmanov, Zh. Eksp. Teor. Fiz. 71, 399 (1976); [transl.: Sov. Phys. JETP 44, 210 (1976)].

[2] S. J. Brodsky, H.-C. Pauli, S. Pinsky, Phys. Reports 301 (1998) 299.

[3] J. Carbonell, B. Desplanques, V.A. Karmanov and J.-F. Mathiot, Phys. Reports 300, 215 (1998).

[4] V.A. Karmanov, J.-F. Mathiot and A.V. Smirnov, Phys. Rev. D77, 085028 (2008).

[5] C.N. Braun et al., Phys. Rev. D8 (1973) 92.

[6] C.J. Bebek et al.,Phys. Rev. D17 (1978) 1693.

[7] H. Ackermann et al., Nucl. Phys. B137 (1978) 294.

[8] P. Brauel et al., Z. Phys. C3 (1979) 101.

[9] S.R. Amendolia et al., Phys. Lett. B178 (1986) 435; Phys. Lett. B277 (1986) 168.

[10] J. Volmer et al., Phys. Rev. Lett. 86 (2001) 1713.

[11] T. Horn et al., Phys. Rev. Lett. 97 (2006) 192001.

[12] V. Tadevosyan et al., Phys. Rev. C75 (2007) 055205.

[13] H.J. Behrend et al., Z. Phys. C49 (1991) 401.

[14] J. Gronberg et al., Phys. Rev. D57 (1998) 33.

[15] B. Aubert et al., Phys. Rev. D80 (2009) 052002.

[16] J.-F. Mathiot, Field theory on the light front, contribution to this conference. 